\title{
Desenvolvimento de um Sistema para Controle de Bordo de Nanossatélite
}

\author{
Alex C. R. Alves* Samaherni M. Dias* \\ Kurios I. P. M. Queiroz* \\ * Laboratório de Automação, Controle e Instrumentação (LACI), \\ UFRN, Natal, RN, Brasil, (alexcarlos27@gmail.com, \\ sama@laci.ufrn.br, kurios@laci.ufrn.br)
}

\begin{abstract}
:
The creation of the nanosatellite standard called Cubesat caused an increase in the number of missions based on small satellites. One of the problems faced during the development of projects with Cubesats is related to the effects caused by the radiation on the electronic components of the satellite. This requires the development of on-board control software to detect and correct errors in orbit so that the mission is not compromised. In this context, this article presents the on-board control system that has been developed for the CONASAT project's Cubesats, a partnership between the Northeast Regional Center (CRN) of the National Institute of Space Research (INPE) and the Federal University of Rio Grande do Norte (UFRN). In addition, some tests and results are also presented, as well as some final considerations.

Resumo: A criação do padrão de nanossatélites chamado Cubesat fez com que ocorresse um aumento no número de missões baseadas em pequenos satélites. Um dos problemas enfrentados durante o desenvolvimento de projetos com Cubesats está relacionado com os efeitos causados pela radiação sobre os componentes eletrônicos do satélite. Isso faz com que seja necessário o desenvolvimento de um software de controle de bordo capaz de detectar e corrigir erros em órbita, de modo que não haja um comprometimento da missão. Diante desse contexto, este artigo apresenta o sistema de controle de bordo que tem sido desenvolvido para os Cubesats do projeto CONASAT, uma parceria entre o Centro Regional do Nordeste (CRN) do Instituto Nacional de Pesquisas Espaciais (INPE) e a Universidade Federal do Rio Grande do Norte (UFRN). Além disso, alguns testes e resultados também são apresentados, bem como, algumas considerações finais.
\end{abstract}

Keywords: Cubesats; OBC; on-board computer; CONASAT; nanosatellites.

Palavras-chaves: Cubesats; OBC; computador de bordo; CONASAT; nanossatélites.

\section{INTRODUÇÃO}

Nos últimos anos, as pesquisas e projetos voltados à área espacial têm sido impulsionados graças ao desenvolvimento de um padrão de nanossatélites (satélites com massa de 1 $\mathrm{kg}$ até $10 \mathrm{~kg}$ ) conhecido como Cubesat.

O surgimento desse padrão possibilitou que diferentes organizações e instituições de ensino desenvolvessem missões direcionadas à ciência espacial, uma vez que os Cubesats se tornaram uma alternativa de baixo custo ao projeto e à construção de pequenos satélites. Contudo, as restrições de tamanho impostas aos nanossatélites acarretaram no aparecimento de desafios no projeto e implementação de missões baseadas em objetos desse porte. Por exemplo, a baixa produção energética dos painéis solares de tamanho reduzido impossibilitava o processamento de bordo. Isso acontecia pelo fato dos componentes eletrônicos, aproximadamente 20 anos atrás, serem menos eficientes do que os encontrados na atualidade. Tal eficiência foi alcançada graças ao avanço da tecnologia móvel, o que permitiu a criação de processadores capazes de operar em baixa potência (Razzaghi, 2012).
Ademais, o ambiente extremamente severo em que os Cubesats são posicionados, quando em órbita, também representa um desafio para pesquisadores e projetistas. A radiação, as mudanças extremas de temperatura e o vácuo, presentes no ambiente espacial, fazem com que sejam necessários alguns cuidados na construção dos componentes e subsistemas do nanossatélite.

Apesar dos desafios enfrentados durante o desenvolvimento de missões com Cubesats, o baixo custo de implementação e de projeto tem feito com que um grande número de objetos desse tipo sejam lançados a cada ano. De acordo com dados apresentados em Kulu (2019), até Janeiro de 2019 foram postos em órbita 1030 Cubesats.

No Brasil, o Centro Regional do Nordeste (CRN) do Instituto Nacional de Pesquisas Espaciais (INPE), localizado em Natal/RN, têm desenvolvido há alguns anos, em parceria com a Universidade Federal do Rio Grande do Norte (UFRN), o projeto intitulado Constelação de Nanossatélites para Coleta de Dados Ambientais (CONASAT).

Os nanossatélites que constituem o projeto CONASAT seguem o padrão Cubesat e são compostos, principal- 
mente, pelo: subsistema de energia elétrica (EPS, do inglês, Electrical Power System); subsistema de determinação e controle de atitude (ADCS, do inglês, Attitude and Determination Control System); subsistema de telemetria e telecomando (TT\&C, do inglês, Telemetry, Tracking and Command); e, subsistema de controle de bordo. Além disso, há também a carga útil, a qual representa o objetivo principal da missão.

Cada um dos subsistemas supracitados desempenha uma função específica no nanossatélite e é de fundamental importância para a missão espacial. Entre eles, destaca-se o subsistema de controle de bordo, cujo elemento principal é o computador de bordo (OBC, do inglês, On-Board Computer), responsável pelo processamento das informações transmitidas ao satélite e pelo gerenciamento e controle dos dados entre os demais subsistemas (Razzaghi, 2012).

Considerando a importância do OBC, o software responsável pelo sistema de controle de bordo deve gerenciar todos os outros subsistemas do Cubesat, bem como gerenciar todo o fluxo de dados entre a estação base em solo e o Cubesat em órbita.

Neste artigo, será apresentada uma breve descrição sobre os Cubesats, considerando o ambiente espacial e as características dos computadores de bordo utilizados por eles. Além disso, o projeto CONASAT será descrito, bem como o sistema de controle de bordo que tem sido desenvolvido para os Cubesats desse projeto.

\section{CUBESATS: UMA VISÃO GERAL}

A criação de padrões de nanossatélites possibilitou o acesso ao espaço por parte de diversas organizações e instituições. Por meio deles, as universidades passaram a desenvolver pesquisas e capacitar estudantes na construção e aplicação de pequenos satélites de maneira mais rápida e barata.

Desse modo, a expansão de atividades espaciais com nanossatélites fez com que um considerável número de objetos desse tipo fossem lançados. Os Cubesats representam grande parte dos lançamentos de pequenos satélites nos últimos anos. Tal padrão contém especificações de dimensões, formato e massa para nano e picossatélites. Ele surgiu, em 1999, como resultado da colaboração entre os professores Jordi Puig-Suari da Universidade Politécnica da Califórnia (Cal Poly), San Luis Bispo, e Bob Twiggs da Universidade de Stanford (CalPoly, 2015). No documento de especificação do padrão, intitulado Cubesat Design Specification (CalPoly, 2015), as dimensões básicas de um Cubesat são apresentadas como sendo 10x10x11,35 cm. Contudo, é comum a descrição do padrão como sendo um cubo com $10 \mathrm{~cm}$ de arestas, com massa de até $1,33 \mathrm{~kg}$, que corresponde a uma unidade $(1 \mathrm{U})$. O acoplamento de várias unidades permite a formação de configurações de satélites maiores como por exemplo $2 \mathrm{U}, 3 \mathrm{U}, 6 \mathrm{U}$ e $8 \mathrm{U}$.

O programa Cubesat foi criado com o intuito de reduzir o custo e o tempo de desenvolvimento de picossatélites (CalPoly, 2015). As diversas configurações possíveis e o consequente aumento na massa dos Cubesats fez com que eles passassem a ser classificados também como nanossatélites.

\subsection{Cubesats no Ambiente Espacial}

O ambiente espacial é um lugar perigoso para satélites uma vez que os expõe à radiação eletromagnética, fluxos densos de plasma, espécies altamente reativas e densidades de gás neutro variável (Anderson and Mitchell, 2005).

Em Lumbwe (2013), são descritos alguns dos distúrbios aos quais estão sujeitos os Cubesats em ambiente espacial. Entre eles, tem-se: arrasto atmosférico e oxigênio atômico; ventos solares e erupções solares; ondas eletromagnéticas em espaço livre; exposição de componentes semicondutores à radiação e mudanças extremas de temperatura; e, detritos espaciais.

Os principais efeitos da radiação sobre os componentes eletrônicos dos Cubesats são a Dose Total Ionizante (TID, do inglês, Total Ionizing Dose) e Efeitos de Evento Único (SEE, do inglês, Single Event Effects). De acordo com LaBel et al. (1996) as principais fontes de partículas energizadas que causam esses efeitos são: prótons e elétrons presos nos cinturões de Van Allen; prótons de raio cósmico e íons pesados; e, prótons e íons pesados proveniente de erupções solares.

O efeito TID é consequência do acúmulo de energia depositada em um material durante um longo período de tempo, o que causa uma degradação dos componentes eletrônicos. São exemplos de efeitos causados por TID: variações na corrente de fuga, tensão de limiar e falhas funcionais (LaBel et al., 1996).

Quando energia suficiente é depositada em um dispositivo por meio do impacto de um único íon, diz-se que ocorreu um SEE. Os efeitos desse tipo de evento podem ser divididos em duas categorias principais: erros permanentes (hard errors) e erros não permanentes (soft errors). Um exemplo de hard error é o chamado SEL (em inglês, Single Event Latchup), que envolve elementos parasitas do circuito e pode ser potencialmente destrutivo. Durante um SEL, a corrente do dispositivo excede os limites especificados e, a fim de corrigir o problema, é preciso reiniciar a energia do aparelho (LaBel et al., 1996).

Um tipo de soft error é o chamado SEU (em inglês, Single Event Upset), o qual é caracterizado por um pulso transitório ou bitflip, atingindo elementos de memória como latchs e registradores. Esse tipo de erro pode ser corrigido ao reiniciar o dispositivo ou por meio da reescrita de dados (LaBel et al., 1996).

\subsection{Computadores de Bordo em Cubesats}

O computador de bordo é o elemento central em um Cubesat. Por meio da interação com todos os subsistemas e carga útil do satélite, o OBC fornece serviços como comando e manipulação de dados, monitoramento de condições internas e marcação de tempo (Heunis, 2014).

O subsistema de controle de bordo é comumente considerado como Subsistema de Comandos e Manipulação de Dados (CDHS, do inglês, Command and Data Handling System). Segundo Wertz and Larson (1999), o CDHS tem como funções principais: recepção, validação, decodificação e distribuição de comandos aos outros subsistemas do 
satélite; e, coleta, processamento e formatação de dados a serem enviados à estação terrestre.

Além disso, algumas características que o OBC deve possuir são: robustez mecânica para suportar as vibrações no momento de lançamento; resistência às condições eletromagnéticas; resistência às condições de temperatura; resistência às altas doses de radiação provenientes de partículas energizadas em órbita; e, baixo consumo de energia (Eickhoff, 2011).

Com o objetivo de reduzir os efeitos da radiação sobre o OBC, algumas técnicas de tolerância a falhas podem ser utilizadas. A implementação dessas técnicas pode ocorrer tanto em hardware quanto em software.

Em hardware, são utilizadas técnicas como: redundância fria, redundância quente ou redundância de módulo triplo. Nesse tipo de abordagem são usados elementos redundantes capazes de substituir componentes defeituosos. Por exemplo, a utilização de dois processadores em um mesmo OBC permite que ambos executem as mesmas operações de forma sincronizada. Quando os valores de saída diferem, são detectados erros que podem ser do tipo SEU. Como forma de correção do erro, o sistema pode reiniciar (Razzaghi, 2012).

No que se refere às técnicas de tolerância a falhas por software, um dos métodos aplicados consiste no desenvolvimento de uma arquitetura modular. Nesse método, o sistema é desenvolvido com uma baixa dependência entre os módulos, o que aumenta a tolerância a falhas, uma vez que os erros em um único módulo não se propagam por todo o sistema (Heunis, 2014).

\section{O PROJETO CONASAT}

O projeto CONASAT foi criado com o objetivo de oferecer uma alternativa ao Sistema Brasileiro de Coleta de Dados Ambientais (SBCDA), uma vez que os satélites que constituem o sistema se encontram em operação há vários anos e já ultrapassaram a vida útil estimada. Isso faz com que esses satélites estejam sujeitos à ocorrência de falhas operacionais.

Tal projeto tem sido desenvolvido pelo INPE/CRN em parceria com a UFRN e tem como proposta a construção, o lançamento e a operação de uma constelação de nanossatélites baseada em Cubesats (Carrara et al., 2014).

Os nanossatélites têm sido projetados com o objetivo de coletar dados ambientais provenientes de plataformas remotas espalhadas ao longo do território brasileiro. Os dados coletados (volume de chuva, temperatura, umidade, poluição do ar, correntes oceânicas e perigos ambientais) devem, então, ser retransmitidos ao centro da missão (Carrara et al., 2014).

Desde o início do projeto, algumas plataformas de desenvolvimento e subsistemas para o padrão Cubesat foram adquiridos pelo INPE/CRN e têm sido utilizados como base para elaboração da missão e do sistema de controle de bordo. Os equipamentos estão instalados no laboratório do projeto CONASAT localizado na sede do INPE/CRN em Natal/RN.

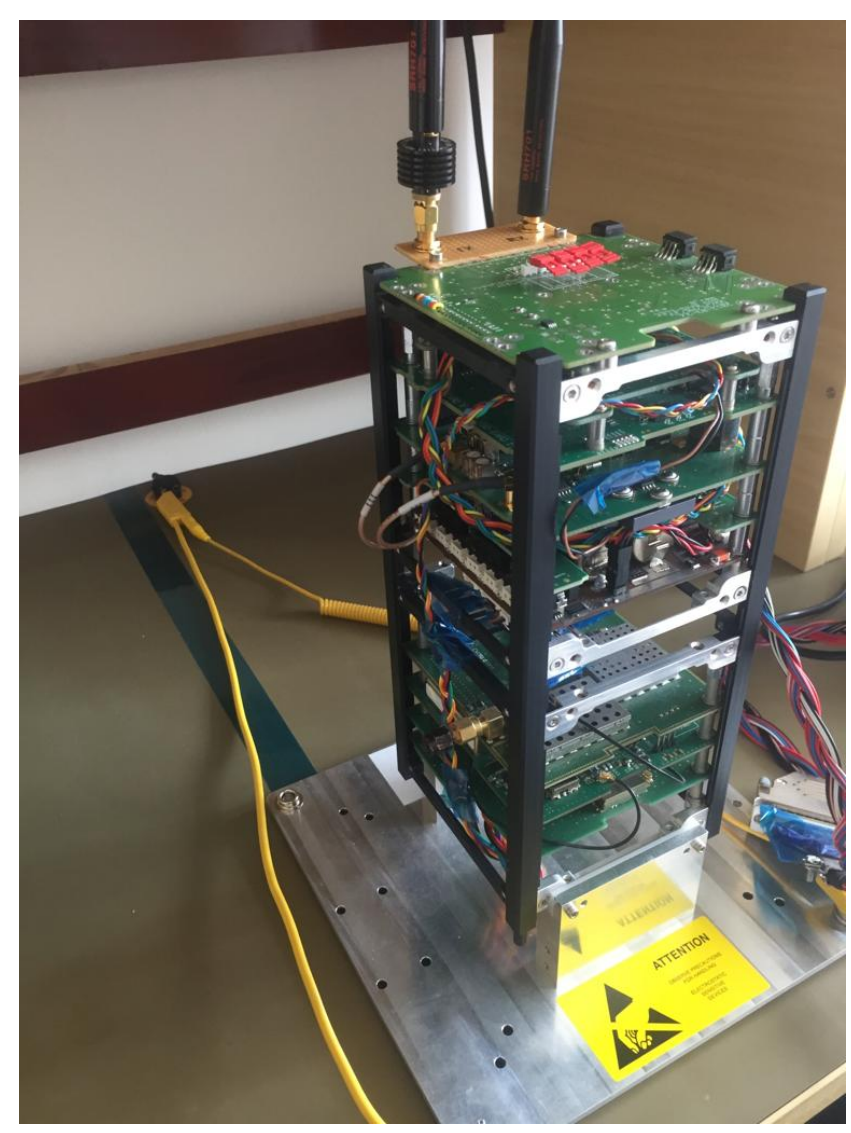

Figura 1. Modelo de Engenharia 2U

Uma dessas plataformas é um modelo de engenharia (réplica do modelo a ser lançado) que segue o padrão Cubesat 2U (Figura 1). Esse modelo é composto por OBC e TT\&C fabricados pela empresa holandesa ISIS (Innovation Solutions in Space). Além disso, há também o subsistema de energia elétrica, que foi fabricado pela empresa dinamarquesa GomSpace. A plataforma permite a integração de diferentes subsistemas e torna possível o desenvolvimento do sistema de controle de bordo, uma vez que oferece componentes para conexão entre o OBC e o computador utilizado para programação e elaboração do software a ser embarcado. Um desses componentes é um adaptador que ao ser conectado ao iOBC oferece uma interface USBSerial para programação e depuração de códigos.

\section{SISTEMA DE CONTROLE DE BORDO}

O sistema de controle de bordo que tem sido desenvolvido é composto pelo computador de bordo, o qual é o nó central de uma arquitetura de processamento descentralizada, e pelo software embarcado.

\subsection{O Computador de Bordo}

O OBC utilizado é chamado iOBC e se encontra acoplado ao modelo de engenharia $2 \mathrm{U}$ descrito anteriormente. Suas características principais são apresentadas na Tabela 1.

O iOBC foi projetado apenas com um processador, o que faz com que a aplicação de técnicas de tolerância a falhas por hardware seja possível por meio do uso de mais de um computador de bordo funcionando de forma redundante. 
Tabela 1. Principais Características do iOBC.

\begin{tabular}{cc} 
Componente & Especificação \\
\hline Processador & ARM9 32-bit 400 MHz \\
Memória Volátil & SDRAM 64 MB \\
Armazenamento de código & Flash NOR 1 MB \\
Armazenamento de Dados & 2 slots de cartão SD (32 GB) \\
Interfaces de Comunicação & $\mathrm{I}^{2} \mathrm{C}, \mathrm{UART}, \mathrm{SPI}$ \\
\hline
\end{tabular}

Contudo, essa abordagem torna o projeto mais caro e requer mais espaço na estrutura do Cubesat. Desse modo, serão adotadas técnicas de tolerância a falhas por software.

A capacidade de processamento desse OBC torna possível a utilização de um sistema operacional em tempo real (RTOS, do inglês, Real Time Operating System) ou de um Linux embarcado.

$\mathrm{O}$ iOBC foi adquirido pelo INPE/CRN com suporte ao sistema FreeRTOS. Contudo, considerando a flexibilidade no desenvolvimento do software, decidiu-se pela utilização de um Linux para sistemas embarcados como sistema operacional. Esse sistema foi instalado em um cartão SD que, posteriormente, foi acoplado a um dos slots disponíveis no iOBC.

\subsection{Arquitetura de Processamento}

Cada um dos subsistemas que constituem os Cubesats do projeto CONASAT apresentam uma unidade de processamento individual. Isso significa que o processamento de dados é realizado por cada subsistema sem que seja necessário o uso do computador de bordo, o qual se encarrega apenas do gerenciamento e do controle desses dados por meio da comunicação pelo barramento $\mathrm{I}^{2} \mathrm{C}$. Esse tipo de processamento caracteriza a arquitetura descentralizada, a qual é ilustrada na Figura 2.

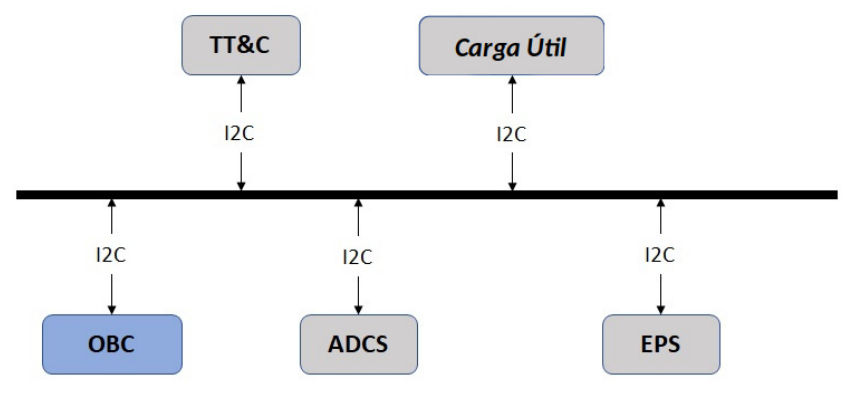

Figura 2. Arquitetura Descentralizada

Uma das vantagens da arquitetura descentralizada é o fato das atividade serem divididas entre várias unidades de processamento. Isso faz com que os pontos de falha sejam isolados e, consequentemente, a falha em um subsistema não compromete a operação do sistema como um todo (Mota, 2017).

\subsection{Software Embarcado}

O software embarcado é dividido em diferentes camadas, as quais executam suas próprias tarefas (Figura 3). A camada de nível mais baixo corresponde às configurações de hardware do computador de bordo. Sobre ela, encontrase a camada de abstração de hardware (HAL, do inglês, Hardware Abstraction Layer), a qual é constituída dos drivers das interfaces de hardware presentes no OBC. O sistema operacional (nesse caso, um Linux embarcado), faz uso das camadas inferiores para possibilitar o gerenciamento do sistema por meio de programas escritos em linguagem de programação de mais alto nível. As aplicações de uma missão são específicas para aquela missão e podem caracterizar códigos que são executados concorrentemente, graças ao sistema operacional. Como exemplo, poderíamos ter duas aplicações em funcionamento: uma encarregada do monitoramento de energia do sistema e outra encarregada de verificar o recebimento de dados de telecomando (enviados pela estação terrestre ao Cubesat).

\begin{tabular}{|c|}
\hline Aplicações de Missão \\
\hline Sistema Operacional \\
\hline HAL \\
\hline Hardware do OBC \\
\hline
\end{tabular}

Figura 3. Camadas do Software

Além disso, com o intuito de minimizar os efeitos da radiação sobre o OBC, foi adotada uma arquitetura modular com técnica de tolerância a falhas. O que significa que os módulos (aplicações) têm sido projetados com baixa dependência, para que erros não se propagem pelo sistema.

\subsection{Inicialização}

O processo de inicialização do sistema começa assim que o OBC é alimentado por uma fonte de energia elétrica (como por exemplo, o EPS). Nesse momento, o bootloader presente na memória Flash NOR carrega o U-Boot (Universal Boot Loader) - bootloader específico para dispositivos embarcados - da memória Flash NOR para a memória SDRAM. O U-Boot, por sua vez, carrega o kernel do sistema operacional, instalado no cartão SD, na SDRAM. Além disso, esse bootloader fornece uma interface de linha de comando (CLI, do inglês, Command-Line Inteface) que permite a depuração e configuração do kernel antes que ele seja carregado na memória SDRAM. Após esse processo, o Linux embarcado é capaz de executar aplicações que tenham sido configuradas para iniciar após o boot do sistema.

\subsection{Modos de Operação}

Os Cubesats do projeto CONASAT têm sido desenvolvidos para que possam operar em seis diferentes modos: Safe; Idle; Detumbling (Det); Nominal (Nom); Payload (Pld); e Control (Ctrl). O controle e gerenciamento desses modos é feito pelo sistema de controle de bordo.

No modo Safe, o satélite deve operar em condições críticas, de maneira que são mantidos em operação apenas os subsistemas essenciais ao funcionamento do sistema (CDHS, EPS). Esse é o modo de operação a ser utilizado assim que o OBC ligar ou quando sofrer um reset. O modo Idle representa a operação padrão do satélite. Nele, o iOBC deve verificar e gerenciar os dados provenientes de sensores e subsistemas, bem como, armazenar informações que podem vir a ser requisitadas pela estação de controle terrestre. O modo Detumbling deve ser utilizado para atenuar o momento angular do satélite. No modo 


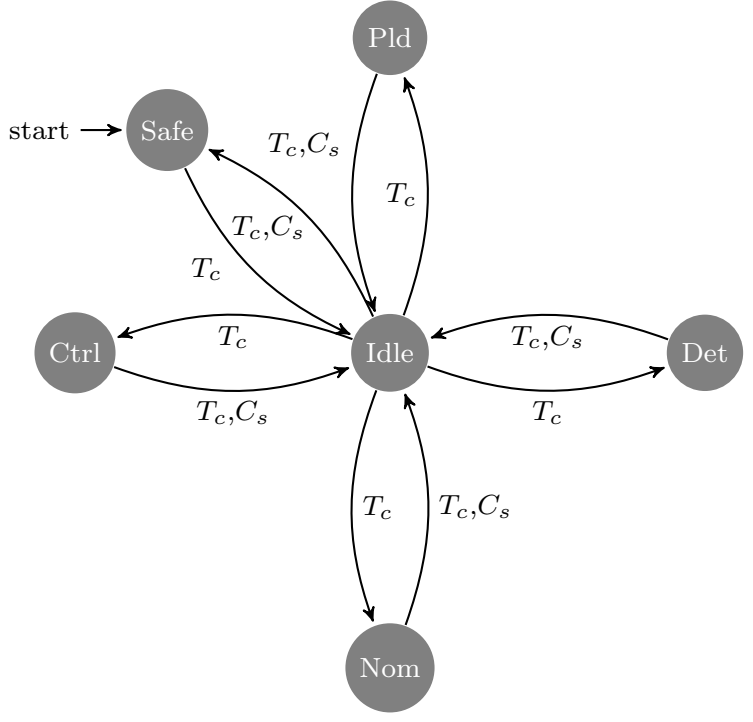

Figura 4. Modos de Operação

Nominal, o OBC deve ativar todos os subsistemas e a carga útil. Sendo assim, haverá uma demanda maior no gerenciamento, controle e armazenamento dos dados. No modo Control, o computador de bordo deve ativar apenas o ADCS, o qual deve efetuar o controle de atitude. O modo Payload deve ser utilizado para que seja ativada apenas a carga útil do satélite. Na Figura 4 pode ser verificada uma representação em máquina de estados dos modos de operação descritos anteriormente. A entrada $T_{c}$ indica a mudança de estado (modo de operação) por meio de um telecomando, o qual é enviado ao satélite pela estação terrestre. A entrada $C_{s}$ caracteriza a aplicação de um comando do sistema que faz com que ocorra uma mudança de estado (modo de operação). Esse comando é gerado quando é verificada uma situação crítica no satélite, como por exemplo, um nível de tensão abaixo do recomendado para o correto funcionamento dos subsistemas.

A partir da máquina de estados, observa-se que, inicialmente, quando o OBC é alimentado, o satélite deve operar no modo Safe, no qual deve permanecer até receber um telecomando para que passe a operar no modo Idle. Esse modo funciona como um estado de transição entre todos os modos de operação. Sendo assim, por meio de telecomandos, o satélite pode passar do modo Idle para qualquer um dos outros modos. Quando o estado atual for diferente dos modos Safe ou Idle, o satélite muda de estado apenas por meio de telecomando e comando do sistema $\left(C_{s}\right)$.

Os modos de operação do satélite são interpretados pelo Linux embarcado como aplicações de missão. Sendo assim, para cada modo descrito, há uma aplicação de software correspondente, que executa funções específicas. As aplicações são gerenciadas por uma aplicação principal de acordo com a máquina de estados apresentada.

\section{TESTES E RESULTADOS PARCIAIS}

Após a inicialização do sistema operacional no iOBC, é possível acessá-lo por meio da interface de comunicação USB-Serial fornecida pelo adaptador citado anteriormente. O diagrama da Figura 5 representa a comunicação entre o
iOBC e o computador pessoal utilizado para programação do sistema de controle de bordo.

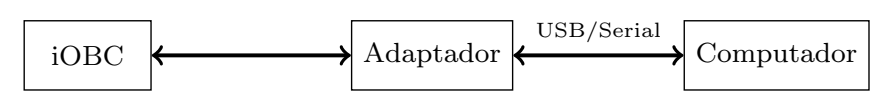

Figura 5. Diagrama de comunicação iOBC-Computador

Os programas desenvolvidos são cross-compilados no computador pessoal e transferidos para o sistema do iOBC por meio da comunicação USB-Serial. Após a transferência, o programa pode ser executado utilizando comandos no terminal Linux.

Com o intuito de validar o funcionamento dos drivers de comunicação $\mathrm{I}^{2} \mathrm{C}$ contidos no Framework instalado, foi testado um programa desenvolvido em linguagem de programação C. Esse programa verificou a escrita e leitura de dados utilizando o barramento $\mathrm{I}^{2} \mathrm{C}$ do iOBC (mestre). Para isso, foi enviada uma mensagem pelo mestre a uma plataforma embarcada externa (escravo). Em seguida, o mestre iniciou o processo de leitura de uma mensagem do escravo. Ao final, verificou-se que não houve perda de dados e que o driver pode ser utilizado também para comunicação com sensores e outros dispositivos que possam vir a ser integrados ao satélite.

Além do teste de driver $\mathrm{I}^{2} \mathrm{C}$, foi realizado um teste da API (Application Programming Interface) do EPS, a qual também faz parte do Framework instalado. Para o teste, foi escrito um programa em $\mathrm{C}$ cujo fluxo de execução é apresentado na Figura 6. Quando o programa é executado, uma função é utilizada para configuração e inicialização da comunicação $\mathrm{I}^{2} \mathrm{C}$. Caso a inicialização ocorra de maneira correta, a execução do programa continua com a solicitação de dados do EPS. Se houver algum erro na inicialização, uma mensagem de erro é exibida no terminal Linux e o programa finaliza sua execução. Quando a função de solicitação executa sem falhas, os dados solicitados são exibidos no terminal, a comunicação $\mathrm{I}^{2} \mathrm{C}$ termina e o programa é finalizado. No entanto, quando a solicitação de dados do EPS falha, uma mensagem de erro é exibida no terminal, a comunicação $\mathrm{I}^{2} \mathrm{C}$ é encerrada e o programa é finalizado.

O resultado da execução do programa descrito anteriormente pode ser verificado na Figura 7. Nesse caso, foram solicitados dados referentes à tensão e temperatura da bateria que compõe o EPS.

\section{CONSIDERAÇÕES FINAIS}

O sistema de controle de bordo para os nanossatélites do projeto CONASAT está em fase de desenvolvimento. Sendo assim, outros testes deverão ser executados a fim de verificar a interação entre os subsistemas que compõem cada Cubesat e o computador de bordo utilizado. Os resultados parciais verificados têm colaborado para o desenvolvimento de aplicações utilizando um Framework específico para Cubesat. Além disso, o Framework tem tornado mais rápida a construção do software embarcado a ser aplicado ao projeto CONASAT. Com a conclusão do projeto de sistema de controle de bordo, espera-se que os resultados possam contribuir com o desenvolvimento de outras missões baseadas em Cubesats. 


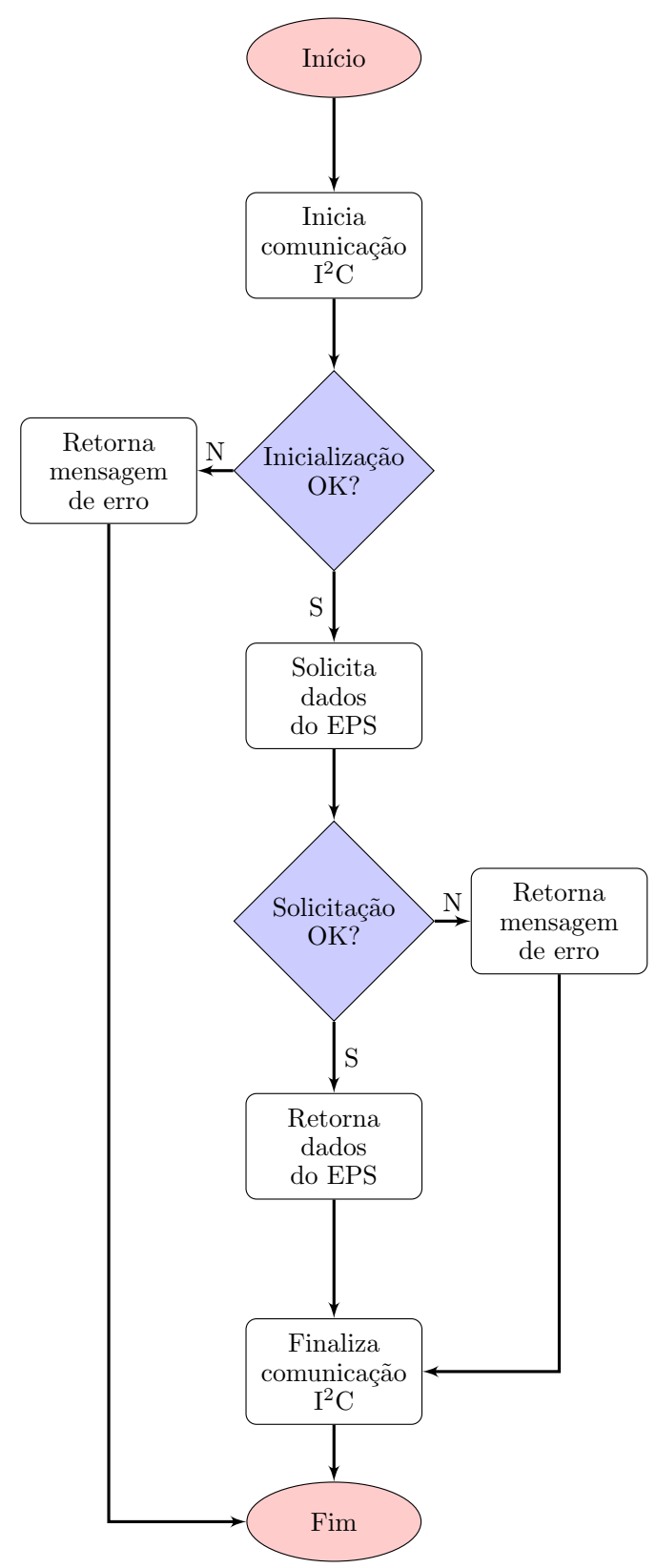

Figura 6. Execução programa EPS

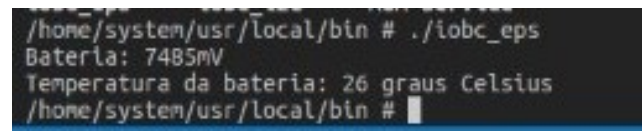

Figura 7. Teste do EPS

\section{AGRADECIMENTOS}

Agradecemos pelo apoio do INPE/CRN e da equipe do projeto CONASAT.

\section{REFERÊNCIAS}

Anderson, B. and Mitchell, D. (2005). The space environment. In V. Pisacane (ed.), Fundamentals of Space Systems. Oxford University Press.

CalPoly (2015). Cubesat design specification rev. 13. [online] Available at: http://www.cubesat.org/s/cds_ rev13_final2.pdf [Accessed 4 May 2019].
Carrara, V., Kuga, H., Bringhenti, P., and de Carvalho, M. (2014). Attitude determination, control and operating modes for conasat cubesats. In 24th International Symposium on Space Flight Dynamics (ISSFD).

Eickhoff, J. (2011). Onboard Computers, Onboard Software and Satellite Operations: An Introduction. Springer Berlin Heidelberg.

Heunis, A. (2014). Design and Inplementation of Generic Flight Software for a Cubesat. Master's thesis, Department Electrical and Electronic Engineering, Stellenbosch University, África do Sul.

Kulu, E. (2019). Nanosatellite database. [online] Available at: http: //www . nanosats . eu [Accessed 4 May 2019].

LaBel, K., Gates, M., Moran, A., Marshall, P., Barth, J., Stassinopoulos, E., Seidleck, C., and Dale, C. (1996). Commercial microelectronics technologies for applications in the satellite radiation environment. In 1996 IEEE Aerospace Applications Conference. Proceedings, volume $1,375-390$.

Lumbwe, L. (2013). Development of an Onboard Computer $(O B C)$ for a Cubesat. Master's thesis, Faculty of Engineering, Cape Peninsula University of Technology.

Mota, D. (2017). Openobc: uma arquitetura de um computador de bordo open source e de baixo custo para o padrão Cubesat. Master's thesis, Centro de Tecnologia, Programa de Pós-Graduação em Engenharia de Teleinformática, Fortaleza, Brasil.

Razzaghi, E. (2012). Design and Qualification of On-Board Computer for Aalto-1 Cubesat. Master's thesis, School of Electrical Engineering, Aalto University, Filândia.

Wertz, J. and Larson, W. (1999). Space Mission Analysis and Design. Microcosm Press, 3 edition. 